PROCEEDINGS OF THE

AMERICAN MATHEMATICAL SOCIETY

Volume 129, Number 7, Pages 1955-1962

S 0002-9939(01)06124-X

Article electronically published on February 15, 2001

\title{
QUASILINEARITY BELOW THE 1ST EIGENVALUE
}

\author{
VICTOR L. SHAPIRO
}

(Communicated by David S. Tartakoff)

\begin{abstract}
This paper establishes the existence of two nontrivial weak solutions for a quasilinear Dirichlet problem below the first eigenvalue via the mountain pass theorem.
\end{abstract}

\section{INTRODUCTION}

Let $\Omega \subset \mathbf{R}^{N}$ be a bounded open connected set, $N \geq 1$. Denote by $D^{\alpha}$ the differential operator

$$
\frac{\partial^{|\alpha|}}{\partial x_{1}^{\alpha_{1}} \cdots \partial x_{N}^{\alpha_{N}}}
$$

where $\alpha=\left(\alpha_{1} \cdots \alpha_{N}\right)$ is a multi-index consisting of nonnegative integers, and $|\alpha|=$ $\sum_{i=1}^{N} \alpha_{i}$ denotes the order of $D^{\alpha}$. In order to write nonlinear partial differential operators in a convenient form, we introduce, as in $\mathrm{Br}$ the vector space $\mathbf{R}^{s_{m}}$ whose elements are of the form

$$
\xi_{m}=\left\{\xi_{\alpha}:|\alpha| \leq m\right\}
$$

where $m$ is a positive integer. For each $u \in W_{0}^{m, p}(\Omega)$, define $\xi_{m}(u)(x)$ to be the vector in $\mathbf{R}^{s_{m}}$ given by

$$
\left\{D^{\alpha}(u(x)):|\alpha| \leq m\right\} \text {. }
$$

(Observe that $D^{(0, \ldots, 0)} u=u$.)

In this paper we study the $2 m$ th-order quasilinear differential operator in generalized divergence form

$$
Q(u)=\sum_{|\alpha| \leq m}(-1)^{|\alpha|} D^{\alpha} A_{\alpha}\left(x, \xi_{m}(u)\right) .
$$

Also we deal with the superlinear differential equation

$$
Q(u)=|u|^{q-2} u+\lambda|u|^{p-2} u
$$

where $\lambda$ is a real number strictly less than the first eigenvalue in $W_{0}^{m, p}(\Omega)$ and $1<p<q$ where $q$ has certain Sobolev restrictions. It will be clear that $u=0$ is a weak solution of $(2)$ in $W_{0}^{m, p}(\Omega)$. We shall show in this paper that under certain

Received by the editors September 7, 1999.

2000 Mathematics Subject Classification. Primary 35J60; Secondary 35J35.

Key words and phrases. Quasilinear variational, first eigenvalue, mountain pass theorem, near $p$-homogeneous. 
further assumptions on $Q$, there are two more nontrivial weak solutions of (2) in $W_{0}^{m, p}(\Omega)$. The result obtained here is to be viewed as a result in the same direction as in [Sh], a previous paper on the first eigenvalue in the space $W_{0}^{m, p}(\Omega)$. It will be clear, also, from the proof to be given that a $g(x, u)$ could be added to the right-hand side of (2), but we will leave these details to the interested reader.

The main thrust in this paper is to use the new idea of near $p$-homogeneity (assumption (A-4) below) in conjunction with variational techniques to obtain a new multiplicity result for a vast new set of equations not handled previously (e.g., examples (28) and (29) below). In particular, in (28) we get an equation involving the mean curvature [GT, p. 357] when $r=1$, which is near $p$-homogeneous but not p-homogeneous.

There is a direct line from the theorem presented here using near $p$-homogeneity through the mountain pass theorem [Ra, p. 7], through the work of Leray and Lions (using (A-3) below, LL, p. 103]) to the variational techniques of Euler and Lagrange.

We will assume that $Q$ has a variational structure in the sense that there exists a potential function $F: \Omega \times \mathbf{R}^{s_{m}} \rightarrow \mathbf{R}$ satisfying

(F-1) The map $x \rightarrow F\left(x, \xi_{m}\right)$ is measurable for each $\xi_{m} \in \mathbf{R}^{s_{m}}$, and the map $x \rightarrow F\left(x, \xi_{m}\right)$ is continuously differentiable for a.e. $x \in \Omega$.

(F-2) There exist constants $p$ and $c_{1}$, with $1<p<\infty$ and $c_{1}>0$, and a nonnegative function $h \in L^{1}(\Omega)$ such that

$$
\left|F\left(x, \xi_{m}\right)\right| \leq h(x)+c_{1}\left|\xi_{m}\right|^{p}
$$

for a.e. $x \in \Omega$ and all $\xi_{m} \in \mathbf{R}^{s_{m}}$.

(F-3) $F(x, 0)=0$ for a.e. $x \in \Omega$, and for each $\alpha$, with $0 \leq|\alpha| \leq m$,

$$
\frac{\partial F}{\partial \xi_{\alpha}}\left(x, \xi_{m}\right)=A_{\alpha}\left(x, \xi_{m}\right) \text { for }\left(x, \xi_{m}\right) \in \Omega \times \mathbf{R}^{s_{m}}
$$

The functions $A_{\alpha}: \Omega \times \mathbf{R}^{s_{m}} \rightarrow \mathbf{R}$ defined in (F-3) above will be assumed to satisfy the Caratheodory conditions (i.e., $A_{\alpha}\left(x, \xi_{m}\right)$ is measurable in $x$ for all $\xi_{m} \in \mathbf{R}^{s_{m}}$ and is continuous in $\xi_{m}$ for a.e. $x \in \Omega$ ) as well as the the following four conditions:

(A-1) There exists a constant $c_{2}$, with $c_{2}>0$, and a nonnegative function $\tilde{h} \in$ $L^{p^{\prime}}(\Omega)$, where $p^{\prime}=p /(p-1)$ and $p$ is as in (F-2) above, such that

$$
\left|A_{\alpha}\left(x, \xi_{m}\right)\right| \leq \tilde{h}(x)+c_{2}\left|\xi_{m}\right|^{p-1}, 0 \leq|\alpha| \leq m,
$$

for a.e. $x \in \Omega$ and all $\xi_{m} \in \mathbf{R}^{s_{m}}$.

(A-2) (Ellipticity) There exists a positive constant $c_{0}$ such that

$$
\sum_{|\alpha| \leq m} A_{\alpha}\left(x, \xi_{m}\right) \xi_{\alpha} \geq c_{0}\left\{\sum_{|\alpha|=m}\left|\xi_{\alpha}\right|^{2}\right\}^{p / 2}
$$

for a.e. $x \in \Omega$ and all $\xi_{m} \in \mathbf{R}^{s_{m}}$ where $p$ is as in (F-2).

(A-3) (Monotonicity) Let $\xi_{m}=\left(\eta_{m-1}, \varsigma_{m}\right)$ be the division of $\xi_{m}$ into its $m$ th order component and the corresponding $(m-1)$ st order terms $\eta_{m-1}$; i.e., $\eta_{m-1}=$ $\left\{\xi_{\beta}: 0 \leq|\beta| \leq m-1\right\} \in \mathbf{R}^{s_{m-1}}$, and $\varsigma_{m}=\left\{\xi_{\alpha}:|\alpha|=m\right\}$. Put $A_{\alpha}\left(x, \xi_{m}\right)=$ $A_{\alpha}\left(x, \eta_{m-1}, \varsigma_{m}\right)$. Then for a.e. $x \in \Omega$ and each $\eta_{m-1} \in \mathbf{R}^{s_{m-1}}$,

$$
\sum_{|\alpha|=m}\left[A_{\alpha}\left(x, \eta_{m-1}, \varsigma_{m}\right)-A_{\alpha}\left(x, \eta_{m-1}, \varsigma_{m}^{*}\right)\right]\left(\varsigma_{\alpha}-\varsigma_{\alpha}^{*}\right)>0 \text { for } \varsigma_{m} \neq \varsigma_{m}^{*} .
$$


(A-4) (Near $p$-homogeneity) For $0 \leq|\alpha| \leq m$,

(i) $A_{\alpha}\left(x, t \xi_{m}\right) t \xi_{\alpha} \leq|t|^{p} A_{\alpha}\left(x, \xi_{m}\right) \xi_{\alpha},|t| \geq 1$,

(ii) $A_{\alpha}\left(x, t \xi_{m}\right) t \xi_{\alpha} \geq|t|^{p} A_{\alpha}\left(x, \xi_{m}\right) \xi_{\alpha},|t| \leq 1$,

for $t \in \mathbf{R}$, a.e. $x \in \Omega$, and all $\xi_{m} \in \mathbf{R}^{s_{m}}$ where $p$ is as in (F-2).

We note, in particular, that (A-4)(ii) and the Caratheodory conditions imply that

$$
A_{\alpha}(x, 0)=0 \text { for } 0 \leq|\alpha| \leq m \text { and a.e. } x \in \Omega .
$$

In the concluding section of this paper (i.e., $\S 4$ ), we shall give three examples of a $Q$ defined by (1) which meets (F-1)-(F-3) and (A-1)-(A-4) above.

We define the following semilinear Dirichlet form:

$$
\mathcal{Q}(u, v)=\sum_{|\alpha| \leq m} \int_{\Omega} A_{\alpha}\left(x, \xi_{m}(u)\right) D^{\alpha} v \forall u, v \in W_{0}^{m, p}(\Omega) .
$$

In view of $(\mathrm{A}-1)$ we see that $\mathcal{Q}$ is well defined on $W_{0}^{m, p}(\Omega) \times W_{0}^{m, p}(\Omega)$.

Observe that by the definition of $\mathcal{Q}$ in (3) and (A-2) we get

$$
\mathcal{Q}(u, u) \geq c_{0} \int_{\Omega}\left\{\sum_{|\alpha|=m}\left|D^{\alpha} u\right|^{2}\right\}^{p / 2}
$$

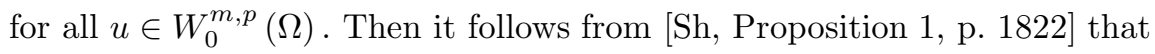

$$
\liminf _{\|u\|_{L^{p}} \rightarrow \infty} \frac{\mathcal{Q}(u, u)}{\|u\|_{L^{p}}^{p}}<\infty
$$

So we define as in [Sh, p. 1821]

$$
\lambda_{1}=\liminf _{\|u\|_{L^{p}} \rightarrow \infty} \frac{\mathcal{Q}(u, u)}{\|u\|_{L^{p}}^{p}} .
$$

We will establish the following result where $\tilde{p}=N p /(N-m p)$ if $m p<N$.

Theorem. Let $1<p<\infty$ and let $\Omega$ be an open bounded connected set. Assume (F-1)-(F-3), and suppose $Q(u)$ is given by (1) where $A_{\alpha}\left(x, \xi_{m}\right)$ satisfies (A-1)(A-4) for $0 \leq|\alpha| \leq m$. Suppose furthermore $\lambda<\lambda_{1}$. Also let $q$ be such that $p<q<\tilde{p}$ for $m p<N$ with $p<q<\infty$ for $m p \geq N$. Then there are at least two nontrivial weak solutions of $(2)$ in $W_{0}^{m, p}(\Omega)$.

We observe that if $v \in W_{0}^{m, p}(\Omega)$ by the Sobolev imbedding theorem [Ad, p. 98], $v \in L^{q}(\Omega)$ where $q$ is as stated in the theorem. Hence, if $u$ also is in $W_{0}^{m, p}(\Omega)$, then $|u|^{q-2} u v$ is in $L^{1}(\Omega)$. Therefore, we say $u$ is a weak solution of equation (2) in $W_{0}^{m, p}(\Omega)$ if

$$
\mathcal{Q}(u, v)=\int_{\Omega}\left[|u|^{q-2} u+\lambda|u|^{p-2} u\right] v \forall v \in W_{0}^{m, p}(\Omega) .
$$




\section{Fundamental Lemmas}

We shall make use of the mountain pass theorem [Ra, p. 7], henceforth designated by (MPT), to establish our theorem. Let $F$ be given as in $(\mathrm{F}-1)-(\mathrm{F}-3)$. Define a functional $\mathcal{I}: W_{0}^{m, p}(\Omega) \rightarrow \mathbf{R}$ by

$$
\mathcal{I}(u)=\int_{\Omega}\left[F\left(x, \xi_{m}(u)\right)-q^{-1}|u|^{q}-\lambda|u|^{p} / p\right] .
$$

Throughout this paper we will use the norm in $W_{0}^{m, p}(\Omega)$ given by

$$
\|u\|_{m, p}^{p}=\sum_{|\alpha| \leq m}\left\|D^{\alpha} u\right\|_{L^{p}}^{p},
$$

where $\|\cdot\|_{L^{p}}$ denotes the $L^{p}$-norm. Also, we note that there are positive constants $c_{3}$ and $c_{4}$ such that

$$
c_{3}\|u\|_{m, p} \leq\left\|\xi_{m}(u)\right\|_{L^{p}} \leq c_{4}\|u\|_{m, p} \forall u \in W_{0}^{m, p}(\Omega)
$$

and from Poincare's inequality (see [GT p. 164]), there is a positive constant $c_{5}$ such that

$$
\left\|\xi_{m}(u)\right\|_{L^{p}}^{p} \leq c_{5} \int_{\Omega}\left\{\sum_{|\alpha|=m}\left|D^{\alpha} u\right|^{2}\right\}^{p / 2} \forall u \in W_{0}^{m, p}(\Omega) .
$$

In order to invoke the (MPT), we shall need the fact that $\mathcal{I}$ defined in (9) satisfies the (PS)-condition, $\mathrm{Ra}$, p. 3]. This will follow from the next two lemmas.

Lemma 1. Assume (F-1)-(F-3), (A-1)-(A-4), $\lambda<\lambda_{1}$, that $p$ and $q$ are as in the theorem, and that $\mathcal{I}$ is given by (9). Suppose that $\left\{u_{n}\right\}$ is a sequence in $W_{0}^{m, p}(\Omega)$ satisfying (i) $\mathcal{I}\left(u_{n}\right)$ is uniformly bounded and (ii) $\mathcal{I}^{\prime}\left(u_{n}\right) \rightarrow 0$ in $W_{0}^{m, p}(\Omega)^{*}$ as $n \rightarrow \infty$. Then there exists a subsequence $\left\{u_{n_{k}}\right\}$ of $\left\{u_{n}\right\}$ which is uniformly bounded in $W_{0}^{m, p}(\Omega)$.

In the above, $W_{0}^{m, p}(\Omega)^{*}$ is understood to be the dual of $W_{0}^{m, p}(\Omega)$, i.e., the space of bounded linear functionals sending $W_{0}^{m, p}(\Omega)$ into R. Also, $\mathcal{I}^{\prime}(u)$ is the Frechet derivative of $\mathcal{I}(u)$.

Lemma 2. Assume (F-1)-(F-3), (A-1)-(A-4), $\lambda<\lambda_{1}$, that $p$ and $q$ are as in the theorem and that $\mathcal{I}$ is given by (9). Suppose that $\left\{u_{n}\right\}$ is a uniformly bounded sequence in $W_{0}^{m, p}(\Omega)$ and is also such that $\mathcal{I}^{\prime}\left(u_{n}\right) \rightarrow 0$ in $W_{0}^{m, p}(\Omega)^{*}$ as $n \rightarrow \infty$. Then $\left\{u_{n}\right\}$ has a convergent subsequence; that is, there exist $u \in W_{0}^{m, p}(\Omega)$ and a subsequence $\left\{u_{n_{k}}\right\}$ satisfying $\lim _{k \rightarrow \infty}\left\|u_{n_{k}}-u\right\|_{m, p}=0$.

Since we are assuming throughout this paper that $\Omega$ is a bounded open connected set and that $p$ and $q$ are as in the theorem, it follows from [Ad, p. 144] that $W_{0}^{m, p}(\Omega)$ is compactly imbedded in $L^{q}(\Omega)$. Using this fact, we see that a proof similar to the one given in [RS Lemma 4] (which hinges on (A-3), the monotonicity condition) also gives the proof of Lemma 2 above; so we will not give the proof of Lemma 2 here and will leave the details of the proof of said lemma to the reader.

We now give the proof of Lemma 1. Suppose to the contrary that the conclusion of the lemma is false. Then, we can suppose that

$$
\lim _{n \rightarrow \infty}\left\|u_{n}\right\|_{m, p}=\infty .
$$


We first show that under assumption (13), there exists a subsequence $\left\{u_{n_{k}}\right\}_{k=1}^{\infty}$ and a positive constant $c_{6}$ such that

$$
\left\|u_{n_{k}}\right\|_{m, p} \leq c_{6}\left\|u_{n_{k}}\right\|_{L^{p}} \quad \forall k .
$$

If (14) does not hold, then

$$
\lim _{n \rightarrow \infty}\left\|u_{n}\right\|_{L^{p}} /\left\|u_{n}\right\|_{m, p}=0
$$

Suppose (15) holds. Then it follows from (3), (F-3), (A-1), and Fubini's theorem that

$$
\int_{\Omega} F\left(x, \xi_{m}(u)\right)=\int_{0}^{1} \mathcal{Q}(t u, u) d t \geq \mathcal{Q}(u, u) / p
$$

where we have also used (A-4)(ii).

Next, we observe from (9), (3), (F-3), and (A-1), that

$$
\mathcal{I}^{\prime}(u)(v)=\mathcal{Q}(u, v)-\int_{\Omega}\left[|u|^{q-2} u+\lambda|u|^{p-2} u\right] v \forall v \in W_{0}^{m, p}(\Omega) .
$$

As a consequence, we see from (9), (16), and (17) that

$$
q \mathcal{I}\left(u_{n}\right)-\mathcal{I}^{\prime}\left(u_{n}\right)\left(u_{n}\right) \geq(q / p-1)\left[\mathcal{Q}\left(u_{n}, u_{n}\right)-\lambda\left\|u_{n}\right\|_{L^{p}}^{p}\right] .
$$

So if (15) holds, we see on dividing both sides of this last inequality by $\left\|u_{n}\right\|_{m, p}^{p}$ and using the conditions in the hypothesis in Lemma 1 that

$$
0 \geq \limsup _{n \rightarrow \infty} \mathcal{Q}\left(u_{n}, u_{n}\right) /\left\|u_{n}\right\|_{m, p}^{p} .
$$

But then (4) joined with (18) tells us that

$$
\lim _{n \rightarrow \infty}\left[\int_{\Omega}\left\{\sum_{|\alpha|=m}\left|D^{\alpha} u_{n}\right|^{2}\right\}^{p / 2} /\left\|u_{n}\right\|_{m, p}^{p}\right]=0 .
$$

From (12) this fact in turn gives that

$$
\lim _{n \rightarrow \infty}\left\|\xi_{m}\left(u_{n}\right)\right\|_{L^{p}} /\left\|u_{n}\right\|_{m, p}=0,
$$

which is a direct contradiction of (11). Hence (15) is false, and (14) is indeed true.

Since $1<p<q$, we next obtain from (13) combined with (14) and Holder's inequality that

$$
\lim _{n_{k} \rightarrow \infty}\left\|u_{n_{k}}\right\|_{L^{q}}=\infty .
$$

Likewise, we see from the conditions in the hypothesis of Lemma 1 combined with (13) and (14) that

$$
\lim _{n_{k} \rightarrow \infty}\left[p \mathcal{I}\left(u_{n_{k}}\right)-\mathcal{I}^{\prime}\left(u_{n_{k}}\right)\left(u_{n_{k}}\right)\right] /\left\|u_{n_{k}}\right\|_{L^{p}}=0 .
$$

Next, we observe from (9), (16), and (17) that

$$
p \mathcal{I}\left(u_{n_{k}}\right)-\mathcal{I}^{\prime}\left(u_{n_{k}}\right)\left(u_{n_{k}}\right) \geq(1-p / q) \int_{\Omega}\left|u_{n_{k}}\right|^{q} .
$$

Dividing both sides of this last inquality by $\left\|u_{n_{k}}\right\|_{L^{p}}$ and passing to the limit as $n_{k} \rightarrow \infty$, we obtain from (20) that

$$
\lim _{n_{k} \rightarrow \infty} \int_{\Omega}\left|u_{n_{k}}\right|^{q} /\left\{\int_{\Omega}\left|u_{n_{k}}\right|^{p}\right\}^{1 / p}=0 .
$$


But by Holder's inequality, there is a positive constant $c_{6}$ such that

$$
c_{6}\left\{\int_{\Omega}|u|^{p}\right\}^{1 / p} \leq\left\{\int_{\Omega}|u|^{q}\right\}^{1 / q} \forall u \in W_{0}^{m, p}(\Omega) .
$$

Hence, we infer from (21) and (22) that

$$
\lim _{n_{k} \rightarrow \infty}\left\{\int_{\Omega}\left|u_{n_{k}}\right|^{q}\right\}^{1-1 / q}=0
$$

This last fact is a direct contradiction of (19). Therefore (13) is not true, and there is indeed a subsequence $\left\{u_{n_{k}}\right\}_{k=1}^{\infty}$ that is uniformly bounded in $W_{0}^{m, p}(\Omega)$, which in fact concludes the proof to Lemma 1.

\section{Proof of the Theorem}

Without loss of generality, we see from (2) that from the start we can assume that $\lambda_{1}>0$. We prove the theorem by observing that with $\mathcal{I}(u)$ for $u \in W_{0}^{m, p}(\Omega)$ defined by $(9)$, the Frechet derivative $\mathcal{I}^{\prime}(u)$ evaluated at $v$ (i.e., $\mathcal{I}^{\prime}(u)(v)$ ) is given by (17). Consequently, if $u_{o} \in W_{0}^{m, p}(\Omega)$ is a critical point of $\mathcal{I}$ - that is, if $\mathcal{I}^{\prime}\left(u_{o}\right)(v)=0$ for all $v \in W_{0}^{m, p}(\Omega)$ - it is clear from (17) and (8) that $u_{o}$ constitutes a weak solution of (2) in $W_{0}^{m, p}(\Omega)$. Furthermore, from (F-3) and (9), it follows that $\mathcal{I}(0)=0$. Hence, if we can show that the critical point $u_{o}$ is such that $\mathcal{I}\left(u_{o}\right) \neq 0$, we will have that $u_{o}$ is a nontrivial weak solution of (2). Also, we observe from (A-4)(i) and (ii) on setting $t=-1$ that

$$
-A_{\alpha}\left(x,-\xi_{m}\right) \xi_{\alpha}=A_{\alpha}\left(x, \xi_{m}\right) \xi_{\alpha} \text { for }|\alpha| \leq m .
$$

Hence, $-A_{\alpha}\left(x,-\xi_{m}\right)=A_{\alpha}\left(x, \xi_{m}\right)$ for $|\alpha| \leq m$. Therefore from (3),

$$
\mathcal{Q}(-u, v)=-\mathcal{Q}(u, v) \forall u, v \in W_{0}^{m, p}(\Omega),
$$

and we see from (17) that $\mathcal{I}^{\prime}(-u)(v)=-\mathcal{I}^{\prime}(u)(v) \forall u, v \in W_{0}^{m, p}(\Omega)$. Therefore, if $u_{o}$ is a nontrivial critical point of $\mathcal{I}$, so is $-u_{o}$. As a consequence, to prove the theorem, we need only show the existence of one nontrivial critical point of $\mathcal{I}$. We shall do this by invoking the (MPT), [Ra, p. 7], and showing that the conditions in the hypothesis of the theorem imply that $\mathcal{I}$ satisfies the conditions in the hypothesis of the (MPT) which in turn implies that $\mathcal{I}$ possesses a nontrivial critical point.

To see that $\mathcal{I}$ defined by (9) does indeed satisfy the conditions in the hypothesis of the (MPT), we take for our real Banach space $E=W_{0}^{m, p}(\Omega)$ and observe from (17), (F-1)-(F-3), (A-1) and (A-2), and the compactness of the imbedding of $E$ in $L^{q}(\Omega)$ that $\mathcal{I} \in C^{1}(E, \mathbf{R})$. Also, it follows from Lemmas 1 and 2 that $\mathcal{I}$ satisfies $(\mathrm{PS})$ and from (9) and (F-3) that $\mathcal{I}(0)=0$.

To complete the proof of the theorem, we see from the (MPT) that it remains to prove

$$
\begin{aligned}
& \exists \rho, \gamma>0 \text { such that } \mathcal{I}(u) \geq \gamma \text { for }\|u\|_{m, p}=\rho ; \\
& \exists \psi \in E \text { with }\|\psi\|_{m, p}>\rho \text { such that } I(\psi) \leq 0 .
\end{aligned}
$$

To establish (23), we observe from (A-4) and (3) that

$$
\mathcal{Q}(r u, r u) \leq|r|^{p} \mathcal{Q}(u, u) \text { for }|r| \geq 1 \text { and } u \in E .
$$

Hence, from our definition of $\lambda_{1}$ and (25), it follows that for $u \in E$,

$$
\frac{\mathcal{Q}(u, u)}{\|u\|_{L^{p}}^{p}} \geq \liminf _{r \rightarrow \infty} \frac{\mathcal{Q}(r u, r u)}{\|r u\|_{L^{p}}^{p}} \geq \lambda_{1}
$$


As a consequence of this last set of inequalities with $\lambda_{1}=\lambda+2 \delta$ where $\delta>0$, we obtain from (9) and (16) that

$$
\mathcal{I}(u) \geq \frac{\delta}{p}\|u\|_{L^{p}}^{p}+\frac{\delta}{\lambda_{1} p} \mathcal{Q}(u, u)-\frac{1}{q}\|u\|_{L^{q}}^{q},
$$

and hence from (4), (11), and (12) that

$$
\mathcal{I}(u) \geq \frac{\delta}{p}\|u\|_{L^{p}}^{p}+\frac{\delta c_{0} c_{3}^{p}}{\lambda_{1} p c_{5}}\|u\|_{m, p}^{p}-\frac{1}{q}\|u\|_{L^{q}}^{q} .
$$

Also, we have from the hypothesis on $q$ and from $\left[\mathrm{Ad}\right.$, p. 98] that there exists $c_{7}>0$ such that $\|u\|_{L^{q}} \leq \mathrm{c}_{7}\|u\|_{m, p} \forall u \in W_{0}^{m, p}(\Omega)$. Hence, we obtain from (26) that

$$
\mathcal{I}(u) \geq \frac{\delta}{p}\|u\|_{L^{p}}^{p}+\left[\frac{\delta c_{0} c_{3}^{p}}{\lambda_{1} p c_{5}}-\frac{c_{7}^{q}}{q}\|u\|_{m, p}^{q-p}\right]\|u\|_{m, p}^{p} .
$$

Since $q>p$, it is clear that the expression in brackets in this last inequality is positive for $\|u\|_{m, p}$ sufficiently small, and (23) follows immediately from this observation.

To show that (24) is valid, take $\phi \in E$ such that $\|\phi\|_{L^{q}} \neq 0$. Then it follows from (9), the equality in (16), and (25) that for $r \geq 1$,

$$
\mathcal{I}(r \phi) \leq r^{p} \int_{0}^{1} \mathcal{Q}(t \phi, \phi) d t-r^{q} \int_{\Omega}|\phi|^{q} / q-\lambda r^{p} \int_{\Omega}|\phi|^{p} / p .
$$

Since $\|\phi\|_{L^{q}} \neq 0$ and $p<q$, it is clear from this last inequality that $\lim _{r \rightarrow \infty} \mathcal{I}(r \phi)=$ $-\infty$. Choose $r_{0}>0$ such that $\mathcal{I}\left(r_{0} \phi\right) \leq-1$ and $\left\|r_{0} \phi\right\|_{m, p} \geq 2 \rho$. Set $\psi=r_{0} \phi$. Then $\psi$ satisfies (24), and (24) is indeed valid. Hence all the conditions in the hypothesis of the (MTP) are met. Consequently, $\mathcal{I}$ possesses a nontrivial critical point, and the proof of the theorem is complete.

\section{EXAMPLES}

We conclude this paper by presenting a few examples of quasilinear operators $Q(u)$ for which the theorem obtained here applies. The first example is for $m=1$ with

$$
Q(u)=-\sum_{|\alpha|=1} D^{\alpha}\left[\left(\sum_{|\beta|=1}\left|D^{\beta} u\right|^{2 k}\right)^{(p-2 k) / 2 k}\left(D^{\alpha} u\right)^{2(k-1)} D^{\alpha} u\right]
$$

where $k$ is a positive integer and $p>1$. For this case, the potential function $F: \mathbf{R}^{N+1} \rightarrow \mathbf{R}$ is then

$$
F\left(\xi_{1}\right)=\frac{1}{p}\left(\sum_{|\alpha|=1} \xi_{\alpha}^{2 k}\right)^{p / 2 k} .
$$

The second example is also for $m=1$ with

$$
Q(u)=\sum_{|\alpha|=1}(-1)^{|\alpha|} D^{\alpha}\left[\left|\xi_{1}^{\prime}(u)\right|^{p-2}+\left(1+\left|\xi_{1}^{\prime}(u)\right|^{2}\right)^{-(2-r) / 2}\right] D^{\alpha} u
$$

where $p \geq 2,1 \leq r<2$, and $\left|\xi_{1}^{\prime}(u)\right|^{2}=\sum_{|\alpha|=1}\left|D^{\alpha} u\right|^{2}$. The potential function $F: \mathbf{R}^{N+1} \rightarrow \mathbf{R}$ in this case is

$$
F\left(\xi_{1}\right)=\frac{1}{p}\left(\sum_{|\alpha|=1} \xi_{\alpha}^{2}\right)^{p / 2}+\frac{1}{r}\left(1+\sum_{|\alpha|=1} \xi_{\alpha}^{2}\right)^{r / 2}-\frac{1}{r} .
$$


For our final example, we let $m$ be an arbitrary positive integer and set

$$
Q(u)=\sum_{|\alpha| \leq m}(-1)^{|\alpha|} D^{\alpha}\left[c_{\alpha}\left|D^{\alpha} u\right|^{p-2}+c_{\alpha}^{*} \frac{\left|D^{\alpha} u\right|^{2(k-1)}}{\left(1+\left|D^{\alpha} u\right|^{2 k}\right)^{(2 k-r) / 2 k}}\right] D^{\alpha} u
$$

where $k$ is a positive integer, $1 \leq r<2 k$, and $p \geq 2 k$. Also, $c_{\alpha}>0$ and $c_{\alpha}^{*} \geq 0$ for $|\alpha| \leq m$. The potential function $F: \mathbf{R}^{s_{m}} \rightarrow \mathbf{R}$ is

$$
F\left(\xi_{m}\right)=\sum_{|\alpha| \leq m}\left[c_{\alpha}\left|\xi_{\alpha}\right|^{p} / p+c_{\alpha}^{*}\left(1+\xi_{\alpha}^{2 k}\right)^{r / 2 k} / r\right]-\sum_{|\alpha| \leq m} c_{\alpha}^{*} / r .
$$

In all of the above three cases, it is an easy matter to see that $F$ meets (F-1)(F-3) and that $A_{\alpha}\left(\xi_{m}\right)=\frac{\partial F}{\partial \xi_{\alpha}}\left(\xi_{m}\right)$ satisfies (A-1)-(A-4) where, in particular, for the monotonicity condition (A-3) we make use of [KS, p. 16]. We also observe that the last two examples actually are near $p$-homogeneous and not $p$-homogeneous.

\section{REFERENCES}

[Ad] R. A. Adams, Sobolev Spaces, Academic Press, New York, 1975. MR 56:9247

[Br] F. E. Browder, Existence theorems for nonlinear partial differential equations, Proc. Sympos. Pure Math. Vol. 16, Amer. Math. Soc., Providence, R. I., 1970, pp. 1-60. MR 42:4855

[KS] D. Kinderlehrer and G. Stampacchia, An Introduction to Variational Inequalities and Their Applications, Academic Press, New York (1980). MR 81g:49013

[GT] D. Gilbarg and N. S. Trudinger, Elliptic partial differential equations of second order, 2nd ed., Springer-Verlag, Berlin, 1983. MR 86c:35035

[LL] J. Leray and J.-L. Lions, Quelques résultats de Višik sur les problèmes elliptiques nonlinéaires par les méthodes Minty-Browder, Bull. Soc. Math. France 93 (1965), 97-107. MR 33:2939

[Ra] P. Rabinowitz, Minimax Methods in Critical Point Theory with Applications to Differential Equations, CBMS Regional Conf. Ser. in Math. , No. 65, Amer. Math. Soc., Providence, R. I., 1986. MR 87j:58024

[RS] A. Rumbos and V. L. Shapiro, One-sided resonance for a quasilinear variational problem, Contemporary Math. Vol. 208, Amer. Math. Soc., Providence, R. I., 1997, pp. 277-299. MR 98j:35074

[Sh] V. L. Shapiro, Quasilinear ellipticity and the first eigenvalue, Comm. Partial Differential Equations 16 (1991), 1819-1855. MR 92j:35053

Department of Mathematics, University of California, Riverside, California 925210135

E-mail address: shapiro@math.ucr.edu 